\title{
Qualidade das prescrições de profilaxia para lesão aguda de mucosa gástrica em um hospital universitário no Brasil
}

\author{
Quality of stress ulcer prophylaxis in a universitary hospital in Brazil
}

\author{
Guilherme da Silva Fioramonte ${ }^{1}$, Giovanna Daneluz de Brito ${ }^{1}$, Gustavo Lenci Marques²
}

Fioramonte GS, Brito GD, Marques GL. Qualidade das prescrições de profilaxia para lesão aguda de mucosa gástrica em um hospital universitário no Brasil / Quality of stress ulcer prophylaxis in a universitary hospital in Brazil. Rev Med (São Paulo). 2020 mar.abr.;99(2):122-7.

RESUMO: Objetivos: Ainda que preconizada para pacientes de alto risco de sangramento gastrointestinal alto, a profilaxia para lesão aguda de mucosa gástrica (úlcera de estresse) nem sempre é prescrita corretamente na maioria nos serviços hospitalares. O objetivo deste estudo é avaliar a adequação das prescrições dessa profilaxia em um hospital universitário brasileiro. Métodos: Realizada uma análise transversal de prescrições para pacientes internados em unidades clínicas, cirúrgicas e críticas de um hospital universitário durante 30 dias. Foram excluídas as prescrições de pacientes em uso de protetores gástricos com finalidade diferente da profilática. Os critérios de avaliação da profilaxia para úlcera de estresse foram baseados nas recomendações da base online de dados UpToDate ${ }^{\circledR}$. Resultados: 358 prescrições foram analisadas e, dessas, 17 foram excluídas. Das 341 prescrições incluídas, $205(60,1 \%)$ estavam inadequadas. A principal razão de inadequação foi a sobreprescrição, encontrada em 175 casos $(85,4 \%)$. As inadequações foram maiores em unidades cirúrgicas $(80 \%)$ quando comparadas às unidades clínicas $(54,1 \%)$ e críticas $(52,5 \%)(\mathrm{p}<0,001)$. Conclusões: Foram registradas expressivas taxas de inadequação para a profilaxia de lesão aguda de mucosa gástrica, principalmente devido ao excesso de prescrições. As unidades cirúrgicas foram responsáveis pelas maiores taxas de inadequação. Esse cenário evidencia a escassez de diretrizes científicas, protocolos locais eficientes e conhecimento ou aceitação dos profissionais acerca da profilaxia para lesão aguda de mucosa gástrica.

Descritores: Prevenção de doenças; Úlcera gástrica/prevenção \& controle; Hospitalização; Prescrições; Inibidores da bomba de prótons; Antagonistas dos receptores histamínicos H2.

\begin{abstract}
Aims: Though recommended for patients with high risk for upper gastrointestinal bleeding, stress ulcer prophylaxis is not always correctly prescribed in most hospitals. The objective of this study is to evaluate the adequation of stress ulcer prophylaxis in a universitary hospital. Methods: A cross-sectional analysis of the medical prescriptions of patients hospitalized in clinical, surgical and critical units of a universitary hospital was performed during 30 days. Prescriptions of patients using gastric protectors for a non-prophylactic purpose were excluded. The criteria for stress ulcer prophylaxis evaluation was based on recommendations from UpToDate ${ }^{\circledR}$ online database. Results: 358 prescriptions were analyzed, of which 17 were excluded. Of the 341 prescriptions included for stress ulcer analysis, 205 $(60.1 \%)$ prescriptions were incorrect. Overprescription was the main reason of inadequacy, found in 175 cases $(85.4 \%)$. The inadequacies in the prescriptions of the surgical units $(80 \%)$ were higher when compared to the clinical (54.1\%) and critical units $(52.5 \%)(\mathrm{p}<0.001)$. Conclusions: important rates of incorrect stress ulcer prophylaxis prescriptions were found, mainly due to overprescription. Higher rates of inadequacies were found in surgical units. This scenario highlights the lack of scientific guidelines, efficient local protocols and professionals' knowledge or acceptance regarding stress ulcer prophylaxis.
\end{abstract}

Keywords: Disease prevention; Gastric ulcer/prevention \& control; Hospitalization; Prescriptions; Proton pump inhibitors; Histamine $\mathrm{H} 2$ antagonists.

Instituição: Hospital de Clínicas da Universidade Federal do Paraná.

1. Acadêmicos de Medicina da Universidade Federal do Paraná. ORCID ID: Fioramonte GS - https://orcid.org/0000-0002-8505-1258; Brito GD - https:// orcid.org/0000-0001-7279-9366.

2. Professor adjunto do Departamento de Clínica Médica da Universidade Federal do Paraná e médico cardiologista do Hospital de Clínicas da Universidade Federal do Paraná. Coordenador Adjunto do Curso de Medicina da Pontifícia Universidade Católica do Paraná, Curitiba, Paraná, Brasil. E-mail: gustavolencimarques@gmail.com. ORCID ID: https://orcid.org/0000-0002-6057-0350.

Endereço para correspondência: Guilherme da Silva Fioramonte. R. Nilo Cairo, 524, apto. 03. Centro. Curitiba, PR. CEP: 80060-050. E-mail: guifioramonte@gmail.com. 


\section{INTRODUÇÃO}

Ser internado em qualquer hospital do mundo sofrem danos durante a hospitalização, sendo que metade deles poderiam ter sido evitados ${ }^{1}$.

A lesão aguda de mucosa gástrica (LAMG), ou "úlcera de estresse", é uma condição relacionada à resposta inflamatória e simpática do trauma, queimaduras, choque ou sepse ${ }^{2}$. O conceito de LAMG abrange desde achados endoscópicos incidentais, superficiais e assintomáticos até o sangramento gastrointestinal, seja ele oculto ou evidente ${ }^{3}$. A LAMG acomete principalmente as regiões do corpo e fundo gástrico e parece estar relacionada à resposta inflamatória e simpática ao estresse, ocasionando redução da perfusão esplâncnica local com redução da secreção gástrica de bicarbonato e aumento da secreção de citocinas pró-inflamatórias e catecolaminas. Consequentemente, ocorre destruição da barreira de muco que, associada à redução da motilidade gástrica, leva ao processo erosivo no epitélio do estômago pela ação ácida ${ }^{4}$. Além de aumentar o risco de morte em 1 a 4 vezes, o sangramento do trato gastrointestinal alto parece ser responsável por aumento de 4 a 8 dias no tempo de permanência em unidade intensiva ${ }^{5}$. Entre $0,6-8,5 \%$ dos pacientes críticos apresentam sangramentos intestinais evidentes, podendo chegar a $15 \%$ se a profilaxia com inibidores de bomba de prótons (IBP) não for realizada ${ }^{4}$. Estudos já demonstraram a eficácia da profilaxia de LAMG com protetores gástricos para situações de alto risco ${ }^{6}$, entretanto, no cenário atual, inadequações ainda são marcantes, com evidente excesso de prescrições no cenário nacional e mundial ${ }^{7-9}$.

Por essas razões, suspeita-se que, mesmo em um hospital universitário, a profilaxia para LAMG não esteja em conformidade com as recomendações preconizadas. O objetivo desse estudo é avaliar o nível de adequação das prescrições de profilaxias para LAMG para pacientes internados em um hospital universitário, referência regional em educação médica.

\section{MÉTODOS}

Realizado um estudo analítico transversal das prescrições médicas de profilaxias para LAMG para pacientes maiores de 18 anos que estiveram internados no período de 19 de julho de 2017 a 19 de agosto de 2017 em enfermarias clínicas e cirúrgicas e em unidades críticas de um hospital universitário em Curitiba, Paraná. Foram excluídas do estudo as prescrições que contemplavam protetores gástricos para outra finalidade que não a profilática. Prescrições de pacientes cujas condições clínicas interferiam na indicação de profilaxias também foram excluídas.
Devido à escassez de diretrizes atualizadas e consagradas sobre o assunto, foram considerados critérios de avaliação as recomendações da base de dados online UpToDate ${ }^{\circledR}{ }^{10}$, que reúne informações bem referenciadas e em conformidade com as evidências científicas mais atualizadas. Foram critérios de alto risco e suficientes para indicação da profilaxia:

1) Coagulopatia definida por contagem de plaquetas $<50.000 / \mathrm{m}^{3}$ ou razão normalizada internacional $(\mathrm{RNI})>$ 1,5 ou tempo de tromboplastina parcial ativada $(\mathrm{TTPa})>$ 2 vezes o controle);

2) Ventilação mecânica por mais de 48 horas;

3) Sangramento ou ulcerações no trato gastrointestinal no último ano;

4) Trauma cerebral;

5) Trauma medular;

6) Pacientes queimados;

7) Dois ou mais critérios menores:

a. Sepse;

b. Tempo em unidade de terapia intensiva por mais de uma semana; de 6 dias;

c. Sangramento gastrointestinal oculto por mais

d. Terapia com glicocorticoide (pelo menos $250 \mathrm{mg}$ de hidrocortisona ou equivalente).

Foram consideradas adequadas, quando indicadas, as seguintes prescrições profiláticas para LAMG:

1) Omeprazol $20 \mathrm{mg}$ via oral ou endovenosa, uma vez ao dia;

2) Omeprazol $40 \mathrm{mg}$ via oral, uma vez ao dia;

3) Ranitidina $150 \mathrm{mg}$ via oral, 2 vezes ao dia;

4) Ranitidina $50 \mathrm{mg}$ via endovenosa, 3 vezes ao dia.

O processo de avaliação das prescrições está ilustrado na Figura 1.

O estudo foi aprovado pelo Comitê de Ética em Pesquisa local (aprovado pelo Comitê de Ética em Pesquisa do Hospital de Clínicas da Universidade Federal do Paraná em 22/06/2017. Parecer no 2.131.561) e, antes do início da coleta diária de dados, o médico assistente responsável pela prescrição assinou Termo de Consentimento Livre e Esclarecido. Os dados coletados foram armazenados em planilha do Microsoft Excel. O nível de adequação das profilaxias foi determinado segundo a concordância entre a conduta e o recomendado pelas diretrizes e os resultados obtidos por variáveis qualitativas foram descritos como frequências e percentuais. Para inferência de associação entre duas variáveis qualitativas, foram utilizados os testes qui-quadrado e teste exato de Fischer. A significância estatística considerada foi de $5 \%$. Os dados foram analisados pelo Software R Core Team, versão 3.4.0 ${ }^{11}$. 


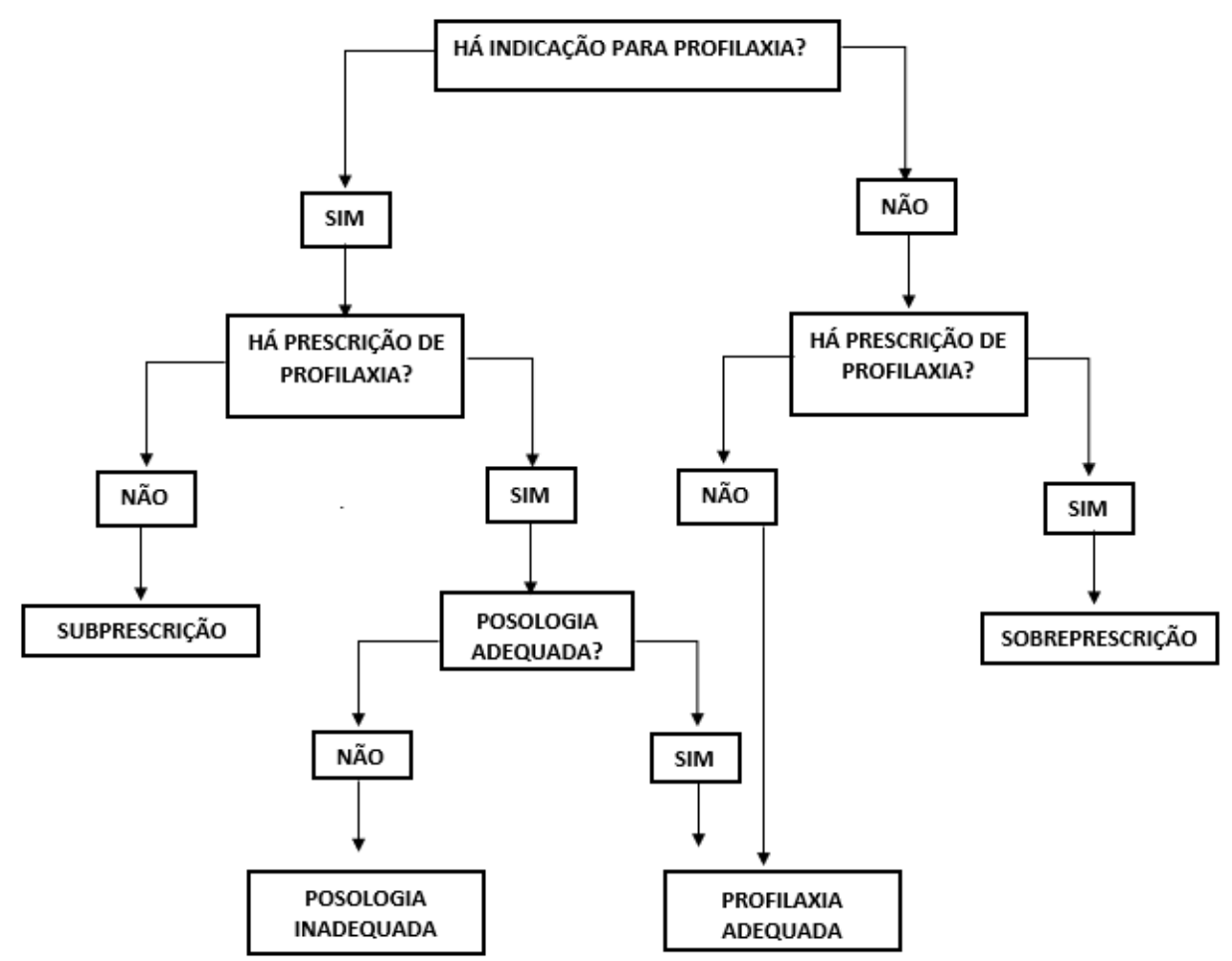

Legenda: O fluxograma ilustra como as prescrições para profilaxia de LAMG foram avaliadas. Quando havia indicação para prescrição, mas essa não era prescrita, a categoria em que essa prescrição foi enquadrada foi a de subprescrição. Quando havia indicação, e ela era prescrita, passava-se para uma nova etapa, que era a análise da posologia. Se fosse inadequada, a categoria foi a intitulada posologia inadequada; se adequada, a precrição foi considerada profilaxia adequada. Por outro lado, quando não havia indicação para profilaxia, mas essa era prescrita, era caracterizada a sobreprescrição; quando não indicada, e não prescrita, foi considerada profilaxia adequada.

Figura 1 - Processo de avaliação das prescrições profiláticas

\section{RESULTADOS}

A Tabela 1 apresenta a distribuição geral da amostra. Foram selecionadas 358 prescrições, das quais 17 (4,75\%) foram excluídas conforme Figura 2. A Figura 3 ilustra os resultados globais do estudo, análise que foi subdividida entre as unidades de internamento e apresentada nas Tabelas 2 e 3 .

Tabela 1 - Distribuição das prescrições selecionadas por unidade de internamento

\begin{tabular}{lcccc}
\hline & \multicolumn{3}{c}{ UNIDADES } & \multirow{2}{*}{ TOTAL } \\
\cline { 2 - 4 } & Clínicas & Cirúrgicas & Críticas & \\
\hline Número de prescrições & $170(47,5 \%)$ & $85(23,7 \%)$ & $103(28,8 \%)$ & $358(100 \%)$ \\
Sexo & & & \\
Masculino & $74(43,5 \%)$ & $45(52,9 \%)$ & $55(53,4 \%)$ & $174(48,6 \%)$ \\
Feminino & $95(55,9 \%)$ & $40(47,1 \%)$ & $48(46,6 \%)$ & $183(51,1 \%)$ \\
Mulher transgênero & $1(0,6 \%)$ & - & - & $1(0,3 \%)$ \\
Idade (anos) & $60,0 \pm 15,8$ & $51,3 \pm 16,6$ & $59,3 \pm 17,1$ & $57,7 \pm 16,7$ \\
\hline
\end{tabular}

NOTA: As unidades clínicas corresponderam a quase metade da amostra, com 170 prescrições. 


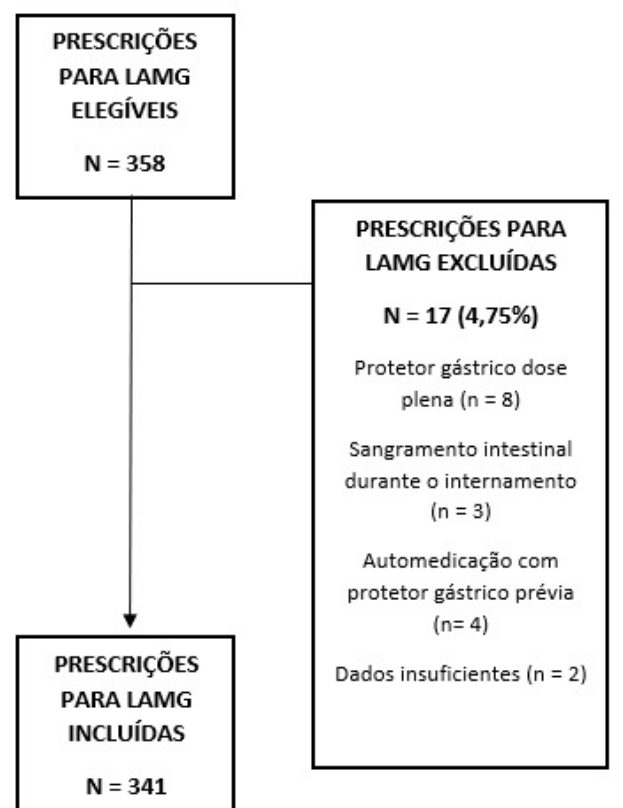

NOTA: Foram selecionadas 358 prescrições para o estudo, sendo 17 delas excluídas, de modo que, ao final, 341 prescrições foram incluídas na análise.

Figura 2 - Relação de prescrições incluídas e excluídas do estudo

A

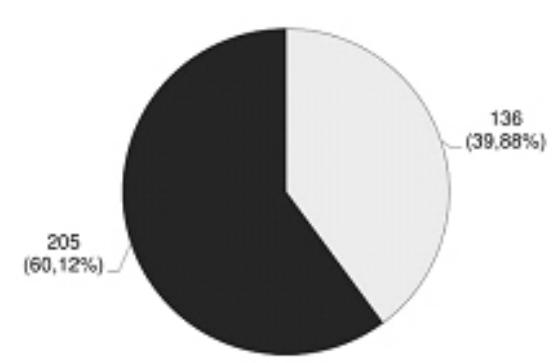

$\square$ Prescriçōes corretas

- Prescrições incorretas
B

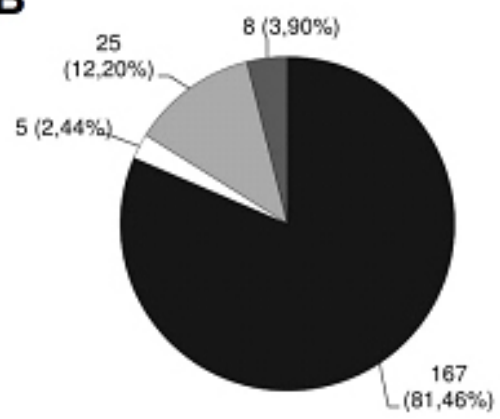

- Sobreprescriçăo isolada

๑ Subprescrição

- Posologia incorreta isolada

- Sobreprescriçāo + posologia incorreta

Figura 3 - Análise geral das prescrições. O gráfico A ilustra a distribuição geral dos níveis de adequação das prescrições de profilaxia para LAMG, e o gráfico B relaciona a distribuição geral dos motivos de inadequação entre as prescrições incorretas para LAMG

Tabela 2 - Adequação das prescrições segundo unidade de internamento

\begin{tabular}{lccc}
\hline & & UNIDADES & Críticas \\
\cline { 2 - 4 } & Clínicas & Cirúrgicas & $47(47,47 \%)$ \\
\hline Prescrições corretas & $72(45,86 \%)$ & $17(20 \%)$ & $52(52,53 \%)$ \\
Prescrições incorretas & $85(54,14 \%)$ & $68(80 \%)$ & 5 \\
\hline
\end{tabular}

NOTA: A maior taxa de inadequação foi encontrada nas unidades cirúrgicas (80\%), seguidas pelas unidades clínicas (54,14\%) e as críticas (52,53\%). Teste exato de Fisher; $\mathrm{p}<0,001$. 
Tabela 3 - Motivos de inadequação das prescrições incorretas segundo unidade de internamento

\begin{tabular}{lccc}
\hline & \multicolumn{3}{c}{ MOTIVO DE INADEQUAÇÃO } \\
\cline { 2 - 4 } & Sobreprescrições & Subprescrições & Posologia incorreta \\
\hline Unidades clínicas & $76(89,41 \%)$ & $2(2,35 \%)$ & $11(12,94 \%)$ \\
Unidades cirúrgicas & $66(97,06 \%)$ & $1(1,47 \%)$ & $1(1,47 \%)$ \\
Unidades críticas & $33(63,46 \%)$ & $2(3,85 \%)$ & $21(40,38 \%)$
\end{tabular}

NOTA: Em todas as unidades, a sobreprescrição foi o motivo de inadequação mais prevalente. As unidades cirúrgicas se destacaram por apresentar as maiores taxas de erro nessa modalidade $(97,06 \%)$, seguidas das unidades clínicas $(89,41 \%)$ e críticas $(63,46 \%)$. As unidades críticas se destacam com relevantes taxas de erro de posologia (40,38\%). Testes qui-quadrado e exato de Fisher; $\mathrm{p}<0,001$

As unidades cirúrgicas apresentaram as maiores taxas de inadequação ( $80 \%$, versus $54,14 \%$ nas unidades clínicas e 52,53\% nas unidades críticas, p<0,001) e a sobreprescrição foi a modalidade de erro mais comum em todas as unidades de internamento.

As prescrições para LAMG com posologia incorreta $(\mathrm{n}=33)$ possuíam a seguinte apresentação: omeprazol $40 \mathrm{mg}$ uma vez ao dia por via endovenosa $(n=20)$; omeprazol 40 $\mathrm{mg}$ a cada 12 horas por via endovenosa $(\mathrm{n}=2)$; ranitidina 50 mg a cada 12 horas por via endovenosa $(n=10)$; prescrição simultânea de omeprazol e ranitidina $(n=1)$.

\section{DISCUSSÃO}

Desde o seu surgimento em meados da década de 1980, os IBP têm sido amplamente utilizados, com crescimento exponencial de suas prescrições. $\mathrm{Na}$ última década, já estiveram entre os dez medicamentos mais consumidos em todo o mundo ${ }^{12}$. Esse fato, associado ao conhecimento defasado dos profissionais da área da saúde sobre as implicações da LAMG e a sua profilaxia, vem criando um cenário global preocupante nas prescrições desses fármacos.

Apesar de dramáticos, os resultados aqui expostos em relação ao uso de protetores gástricos não parecem ser uma exceção. A taxa de erro geral na prescrição de profilaxias para LAMG encontrada nesta análise foi de $60,12 \%$, número compatível com outros estudos existentes na literatura. Parente e colaboradores, em 2003, demonstraram que cerca de $68 \%$ das prescrições para protetores gástricos eram inadequadas em um hospital universitário italiano ${ }^{7}$. Em 2006, Pham et al. ${ }^{8}$ demonstraram que $71 \%$ dos pacientes não críticos do Hospital da Universidade de Michigan recebiam supressores gástricos, mas apenas $10 \%$ deles possuíam indicações aceitáveis para tal. No Brasil o cenário é semelhante e, em 2006, um estudo demonstrou taxa de $71 \%$ de prescrição de protetores gástricos em pacientes com risco intermediário e baixo para LAMG, situação sem respaldo científico claro para indicação de profilaxia. Por outro lado, esse mesmo estudo aponta que $25,7 \%$ dos pacientes de alto risco não recebiam a profilaxia para LAMG, apesar de possuírem indicação para tal ${ }^{9}$. Trata-se de um número bastante distante da taxa de subprescrição aqui apresentada de 2,44\%.

Nesta análise, os resultados mais impressionantes foram encontrados nos internamentos cirúrgicos, com $80 \%$ de inadequações, majoritariamente devido à sobreprescrição, que foi justamente a modalidade de erro mais expressiva em todo o estudo. É possível que a sobreprescrição ocorra devido a popularização crescente do uso dos IBP associada ao desconhecimento da comunidade médica acerca das indicações de profilaxia e efeitos colaterais das medicações. Também é possível a hipótese de que o médico assistente, visando minimizar o risco de LAMG em seus pacientes, possa acabar desconsiderando o ônus ao sistema de saúde que sua sobreprescrição pode causar. Em 2006, o custo adicional relacionado às prescrições inadequadas para LAMG foi calculado em US\$111.791,00 por ano para pacientes não críticos sob cuidados de seis das unidades do complexo hospitalar da University of Michigan Health Service, incluindo pacientes hospitalizados e não hospitalizados ${ }^{13}$. Estudos sobre o impacto financeiro dessa prática no cenário brasileiro são relevantes e devem ser encorajados, principalmente ao se considerar a carência de recursos e investimentos que os hospitais públicos brasileiros enfrentam todos os dias.

A indicação de profilaxia de LAMG ainda é muito debatida na literatura. Apesar dos fatores de risco para essa condição serem bem conhecidos, estudos recentes têm questionado a real eficácia de se usar protetores gástricos para profilaxia de úlcera de estresse, bem como o benefício da sua prescrição frente aos efeitos colaterais dos medicamentos ${ }^{14}$. Os efeitos adversos mais relatados são pneumonia nosocomial e infecção por Clostridium difficile, mas outras reações possíveis incluem má absorção de micronutrientes e nefrite intersticial aguda. A incidência e relevância clínica dessas complicações ainda apresentam dados inconsistentes ${ }^{15-17}$, o que não justifica que a prescrição e protetores gástricos não deva ser uma preocupação do médico hospitalista, visto que a profilaxia ainda é recomendada pela imensa maioria das diretrizes vigentes, com critérios de indicação precisos, como já exposto ${ }^{10,18}$. É preciso ressaltar, porém, que a ausência de uma diretriz consagrada de referência em profilaxia para LAMG gera 
viés de aferição em qualquer metodologia de avaliação de qualidade proposta acerca do tema.

É possível que este estudo tenha sofrido interferência do efeito Hawthorne, caracterizado, neste caso, por uma mudança positiva no padrão de prescrições dos médicos assistentes após aplicação do Termo de Consentimento Livre e Esclarecido. Esse viés, inerente da metodologia, pode ter sido consideravelmente reduzido uma vez que a coleta de dados sempre ocorreu após a realização da prescrição do dia nas enfermarias e unidades críticas. Nesse contexto, levantamos a hipótese de estarmos diante de uma representação amenizada do cenário de prescrições profiláticas. Frente a essa preocupação, estudos futuros com metodologia diferente são oportunos.

Recomenda-se ainda que estudos futuros aprofundem a caracterização desses agravos na população local para melhor definição do cenário vigente. Sugere-se também a ampliação da metodologia para análise de outras entidades clínicas causadoras de danos hospitalares que também são potencialmente evitáveis, como delirium e úlcera de pressão.

\section{CONCLUSÕES}

O presente estudo confirmou a hipótese proposta ao revelar significantes taxas de inadequações de prescrições para profilaxia de LAMG em pacientes internados em um hospital universitário, sendo as maiores inadequações observadas nas unidades cirúrgicas. Fundamenta-se, assim, a necessidade de intervenções que otimizem essa importante prática de segurança aos pacientes hospitalizados. Dessa forma, espera-se que, no futuro, ser internado em qualquer hospital do mundo não seja algo tão arriscado.

Agradecimentos: A nossa escola centenária, a Universidade Federal do Paraná e o Hospital de Clínicas da UFPR, para que continuemos prezando pela excelência no ensino médico.

Os autores declaram não haver conflitos de interesses.

Os autores se responsabilizam pelo conteúdo deste trabalho.

Participação dos autores: Fioramonte GS: revisão bibliográfica, coleta de dados, interpretação dos dados, redação do manuscrito (Resumo/ Abstract, Introdução, Discussão e Referências). Brito GD - revisão bibliográfica, coleta de dados, interpretação dos dados, redação do manuscrito (Resumo/Abstract, Introdução, Métodos). Marques GL - idealização do estudo, delineamento da análise, análise estatística, redação do texto (Introdução, Discussão e Conclusão), revisão final do manuscrito.

\section{REFERÊNCIAS}

1. World Health Organization. 10 Facts on patient safety [cited 2018 Apr 30]. Available from: http://www.who.int/features/factfiles/ patient_safety/en.

2. Cook DJ. Stress ulcer prophylaxis: gastrointestinal bleeding and nosocomial pneumonia. Best evidence synthesis. Scand J Gastroenterol Suppl. 1995;210:48-52. doi: 10.3109/00365529509090271.

3. Plummer MP, Blaser A, Deane AM. Stress ulceration: prevalence, pathology and association with adverse outcomes. Crit Care. 2014;18(2):213. doi: 10.1186/cc13780.

4. Madsen KR, Lorentzen K, Clausen N, Oberg E, Kirkegaard PRC, Maymann-Holler N, et al. Guideline for stress ulcer prophylaxis in the intensive care unit. Dan Med J. 2014;61(3):C4811.

5. Cook DJ, Griffith LE, Walter SD, Guyatt GH, Meade MO, Heyland DK, et al. The attributable mortality and length of intensive care unit stay of clinically important gastrointestinal bleeding in critically ill patients. Crit Care. 2001;5(6):368. doi: 10.1186/cc1071.

6. Cook DJ, Reeve BK, Guyatt GH, Heyland DK, Griffith LE, Buckingham L, et al. Stress ulcer prophylaxis in critically ill patients. Resolving discordant meta-analyses. JAMA. 1996;275(4):308-14. doi: 10.1001/jama.1996.03530280060038.

7. Parente F, Cucino C, Gallus S, Bargiggia S, Greco S, Pastore L, et al. Hospital use of acid-suppressive medications and its fallout on prescribing in general practice: a 1-month survey. Aliment Pharmacol Ther. 2003;17(12):1503-6. doi: 10.1046/j.02692813.2003.01600.x.

8. Pham CQD, Regal RE, Bostwick TR, Knauf KS. Acid suppressive therapy use on an inpatient internal medicine service. Ann Pharmacother. 2006;40(7-8):1261-6. doi: 10.1345/aph.1G703.

9. Machado AS, Teixeira C, Furlanetto L, Tonietto T, Balzano P de C, Vieira SRR, et al. Profilaxia para úlcera de estresse nas unidades de terapia intensiva: estudo observacional multicêntrico. Rev Bras Ter Intensiva. 2006;18(3):229-33. doi: 10.1590/S0103507X2006000300003.

10. Weinhouse GL. Stress ulcer prophylaxis in the intensive care unit. Indications, choosing an agent, efficacy, potential harms, summary and recommendations. UpToDate, 2015 [cited 2016 Nov 07]. Avaliable from: https://www.uptodate.com/contents/ stress-ulcer-prophylaxis-inthe-intensive-care-unit.

11. R Core Team. R: a language and environment for statistical computing. R Foundation for Statistical Computing. Vienna, Austria; 2017. Available from: http://www.r-project.org/.

12. Lindsley CW. The top prescription drugs of 2011 in the United States: antipsychotics and antidepressants once again lead CNS therapeutics. ACS Chem Neurosci. 2012;3(8):630-1. doi: $10.1021 / \operatorname{cn} 3000923$.

13. Heidelbaugh JJ, Inadomi JM. Magnitude and economic impact of inappropriate use of stress ulcer prophylaxis in non-ICU hospitalized patients. Am J Gastroenterol. 2006;101(10):2200-5. doi: 10.1111/j.1572-0241.2006.00839.x.

14. Krag M, Perner A, Wetterslev J, Wise MP, Hylander Møller M. Stress ulcer prophylaxis versus placebo or no prophylaxis in critically ill patients. Intensive Care Med. 2014;40(1):11-22. doi: 10.1007/s00134-013-3125-3.

15. Barkun AN, Bardou M, Pham CQD, Martel M. Proton pump inhibitors vs. histamine 2 receptor antagonists for stress-related mucosal bleeding prophylaxis in critically Ill patients: a metaanalysis. Am J Gastroenterol. 2012;107(4):507-20. doi: 10.1038/ ajg.2011.474.

16. Alhazzani W, Guyatt G, Alshahrani M, Deane AM, Marshall JC, Hall R, et al. Withholding pantoprazole for stress ulcer prophylaxis in critically Ill patients. Crit Care Med. 2017;45(7):1121-9. doi: 10.1097/CCM.0000000000002461.

17. Geevasinga N, Coleman PL, Webster AC, Roger SD. Proton pump inhibitors and acute interstitial nephritis. Clin Gastroenterol Hepatol. 2006;4(5):597-604. doi: 10.1016/j.cgh.2005.11.004.

18. ASHP Therapeutic Guidelines on Stress Ulcer Prophylaxis. ASHP Commission on Therapeutics and approved by the ASHP board of directors on November 14, 1998. Am J Health Syst Pharm. 1999;56(4):347-79. doi: 10.1093/ajhp/56.4.347.

Recebido: 30.10 .2019

Aceito: 12.03 .2020 